ANDRIY TANASIYCHUK

Facoltà di Diritto Canonico San Pio X, Venezia

\title{
"PONTIFICIA PRAECEPTA DE CLERO UXORATO ORIENTALI" E LA QUESTIONE DEL CELIBATO PRESSO LE CHIESE ORIENTALI CATTOLICHE
}

Sommario: Introduzione. - 1. Documenti della Sede Apostolica e la loro validità. - 2. Estensione territoriale e personale dei Decreti 1929-1930. - 3. Le espressioni "regioni orientali" e "territori del rito orientale". - 4. La nozione dell' "Ordinariato per fedeli orientali". - 5. La situazione attuale. - 6. Le questioni da risolvere. - Conclusione.

\section{Introduzione}

Il 14 giugno 2014 nella gazzetta ufficiale degli Acta Apostolicae Sedis è stato pubblicato un Documento ${ }^{1}$ da parte della Congregazione per le Chiese Orientali con il quale sono state introdotte notevoli modifiche circa le prescrizioni canoniche vigenti riguardanti eventuali possibilità di conferire l'ordinazione sacra del diaconato e del presbiterato ai candidati uxorati appartenenti alle circoscrizioni ecclesiastiche delle Chiese Orientali Cattoliche costituite in diaspora.

Occorre sottolineare che anche le norme odierne della legislazione comune orientale $(\mathrm{CCEO})$ prevedono questa possibilità però in un paragrafo è indicato che oltre alle norme comuni di tutte le Chiese Orientali Cattoliche possono esistere anche altre disposizioni canoniche in riferimento ad una sola Chiesa sui iuris (diritto particolare) o norme speciali stabilite dalla Sede Apostolica (cfr. can.

\footnotetext{
${ }^{1}$ Congregatio pro Ecclesils Orientalibus, Pontificia Praecepta de Clero Uxorato Orientali, 14 giugno 2014, AAS 106 (2014) 496-499.
} 
$758 \$ 3$ CCEO). Inoltre il Documento vaticano del 2014 fa notare che attualmente quasi tutte le Chiese Orientali Cattoliche godono di questo diritto, cioè possono ordinare i candidati sposati. Eccezione si trova presso le Chiese Siro-Malabarese e Siro-Malancarese che si sono pronunciate in questa materia, cioè hanno stabilito nel proprio diritto particolare che il candidato al diaconato e al presbiterato deve essere celibe ${ }^{2}$. E necessario dire anche che ci sono altre Chiese sui iuris di tradizione bizantina, come per esempio la Chiesa Bulgara e la Chiesa Albanese, che hanno deciso di conferire l'ordinazione diaconale e presbiterale esclusivamente ai candidati di stato celibe ${ }^{3}$.

\section{Documenti della Sede Apostolica e la loro validità}

Le norme particolari di alcune Chiese Orientali Cattoliche in questa materia si considerano attuali perché sono state emanate dopo la promulgazione (1990) ed entrata in vigore (1991) del Codice orientale, invece i vari documenti della Sede Apostolica in merito appartengono alla categoria dei documenti più datati.

La questione del servizio sacerdotale del clero uxorato e l'ordinazione dei candidati sposati presso i fedeli orientali cattolici, cioè ucraini e ruteni, che sono immigrati, dalle regioni sub-carpatiche e dalla parte ovest del territorio ucraino attuale, nell'America di Nord (Stati Uniti e Canada) prima del 1900, furono oggetto di studio da

${ }^{2}$ Cfr. P. Gefaell, Il celibato sacerdotale nelle Chiese orientali: storia, presente, avvenire, in: Il celibato sacerdotale: teologia e vita, Atti del XIV convegno internazionale della Facoltà di Teologia, Pontificia Università della Santa Croce, Roma, 4-5 marzo 2010, a cura di L. Touze, J.M. Arroyo, Roma 2012, 135-156. L'Autore mostra come esempio un decreto della Chiesa Siro-Malabarese - Decree On Clerics, $1 / 1 / 1999$, art. $14 \$ 2$, che è stato pubblicato in Syro-malabar Church since the Eastern Code. Festschrift in Honour of Prof. Georg Nedungatt S.J., An Evaluation and Future Prospects. Particular Laws, Statutes, Decrees, Bibliography, a cura di F. Eluvathingal, Mar Thoma Yogam, Roma 2002, 294. Più tardi questo decreto è entrato a far parte del diritto particolare di questa Chiesa sui iuris, Particular Laws of the Syro-Malabar Church in Bulletin of the Syro-Malabar Major Archiepiscopal Church, art. $34 \$ 2,11(2003) 1$.

${ }^{3}$ Cfr. D. Salachas, N. Nitkiewicz, Rapporti interecclesiali tra cattolici orientali e latini: Sussidio canonico-pastorale, Roma 2007, 47. 
parte di vari dicasteri della Curia Romana. Tali studi hanno portato in breve tempo all'emanazione in merito di tre documenti della Sede Apostolica (1929-1930) ${ }^{4}$.

La legislazione canonica odierna, dopo aver iniziato ad obbligare i fedeli orientali cattolici, da una parte ha abrogato tutte le leggi precedenti, comuni e particolari, che erano contrarie alle proprie norme attuali, dall'altra ha lasciato in vigore le norme precedenti (cfr. can. 6,1 CCEO) solo per quelle materie che nella nuova legislazione della Chiesa cattolica sono state ordinate parzialmente. Le norme rimaste in vigore dopo 1991 sono i tre Decreti della Sede Apostolica, ai quali si riferisce il Documento attuale della Congregazione per le Chiese Orientali del 14 giugno 2014, e che si fondano su altri decreti vaticani che riguardavano tale materia e che sono stati emanati nel 1890 e nel $1913^{5}$.

I decreti della Congregazione orientale (1929-1930) proibivano ai sacerdoti uxorati orientali cattolici di esercitare il proprio ministero su alcuni territori nazionali. Si trattava dei territori dell'America Nord e Sud, Canada e Australia. Inoltre in uno di questi Decreti fu prescritto che l'ammissione al seminario e la successiva ordinazione potevano essere esclusivamente solo per i candidati non sposati. La conseguenza negativa di questi tre Decreti fu il fatto che oltre duecentomila fedeli orientali cattolici che provenivano dall'ovest dell'Ucraina sono passati alla Chiesa ortodossa.

\footnotetext{
${ }^{4}$ Sacra Congregatio pro Ecclesiae Orientali, Decreto Cum data fuerit, 1 marzo 1929, AAS 21(1929) 152-159; SaCra Congregatio pro ECClesiae OrienTALI, Decreto Qua sollerti, 23 dicembre 1929, AAS 22(1930) 99-105; SACRA Congregatio pro Ecclesiae Orientali, Decreto Graeci-Rutheni, 24 maggio 1930, AAS 22(1930) 346-354.

${ }^{5}$ Cfr. D. Salachas, L. Sabbarese, Chierici e ministro sacro nel Codice latino e orientale. Prospettive interecclesiali, Città del Vaticano 2004, 344-345. Gli autori di questo contributo scientifico nella nota 157 hanno sottolineato che subito dopo l'entrata in vigore del CCEO esisteva una certa discussione tra i canonisti circa l'obbligatorietà di questi documenti del passato. Però, con la risposta ufficiale della Congregazione per le Chiese Orientali che ha confermato in vigore i documenti emanati in passato del 1929-1930, terminò questa discussione.
} 


\section{Estensione territoriale e personale dei Decreti 1929-1930}

I Decreti della Curia Romana del 1929 e 1930 che obbligavano i fedeli greco-cattolici residenti nel continente Americano e in Australia, così come è stato detto, non solo continuavano a conservare la propria efficacia giuridica dopo l'entrata in vigore del Codice orientale, ma successivamente sono sono stati "modificati". Le successive modifiche riguardavano due questioni. La prima si riferiva all'estensione della forza obbligatoria di questi documenti su nuovi territori, che all'inizio non furono menzionati nei Decreti, mentre la seconda modifica trattava dell'applicabilità di questi documenti non solo ai fedeli specificati nei documenti, ma anche verso tutti i fedeli orientali cattolici residenti in diaspora.

Poiché manca una conferma ufficiale di queste affermazioni nel Bollettino della Sede Apostolica (AAS), su tale questione si fa riferimento alla riflessione scientifica offerta da Dimitrio Salachas, Krzysztof Nitkiewicz e Antoine Fleyfel ${ }^{6}$.

I primi due autori sostengono che le prescrizioni posteriori in questa materia, emanati dalla Sede Apostolica, non solo confermavano la validità dei Decreti 1929 e 1930, ma anche allargavano l'ambito della loro obbligatorietà anche su altri territori che prima non facevano parte delle così dette regioni orientali. Inoltre tali prescrizioni proibivano di introdurre modifiche su questi argomenti senza previa consultazione con la Conferenza Episcopale di una determinata nazione e senza il permesso esplicito dalla Sede Apostolica.

Invece Antoine Fleyfel nell'articolo dedicato alla presenza del clero uxorato cattolico in Europa Occidentale ${ }^{7}$, fa riferimento ad un certo Documento della Congregazione per le Chiese Orientali del 9 giugno 1992. Dal contesto dell'articolo si può capire che questo Documento era una risposta data all'Ordinario per i fedeli orientali in Francia.

\footnotetext{
${ }^{6}$ Cfr.D. Salachas, K. Nitkiewicz, Rapporti interecclesiali tra cattolici orientali e latini: Sussidio canonico-pastorale, Roma 2007, 47.

${ }^{7}$ Cfr. A. Fley fel, Quelques réflexions sur la présence en Occident de prétres catholiques orientaux mariés, Istina 54(2009), 421-422; P. GEFAELL, Il celibato sacerdotale nelle Chiese orientali: storia, presente, avvenire..., 143-144.
} 
L'Autore dimostra chiaramente le modifiche avvenute nella normativa in relazione alla presenza in Europa occidentale dei sacerdoti sposati appartenenti alle Chiese Orientali Cattoliche. In tale Documento veniva deciso non solo di non introdurre alcune modifiche circa gli argomenti trattati dallo stesso Dicastero nei propri Documenti precedenti (1929 e 1930), ma veniva anche introdotta una nuova prescrizione con la quale si estendeva la forza obbligatoria dei Documenti del 1929 e del 1930 su altri territori degli Stati occidentali e nei territori dove prevale la tradizione del rito latino. L'Autore nell'articolo sottolinea anche un'altra particolarità di questo Documento, che cioè queste decisioni della Congregazione per le Chiese Orientali hanno ottenuto l'approvazione del Romano Pontefice il 9 luglio 1992.

Perciò questo Documento della Congregazione per le Chiese Orientali del 1992 ha esteso la forza obbligante dei Documenti precedenti su altri territori nazionali e ha iniziato ad obbligare tutti i fedeli orientali cattolici che si trovavano fuori dalle proprie regioni orientali.

\section{Le espressioni "regioni orientali" e "territori del rito orientale"}

Una attenzione particolare merita l'espressione regioni orientali che viene menzionata nel Documento della Congregazione per le Chiese Orientali del 2014. In questo Documento si sottolinea che le prescrizioni amministrative previste nei Decreti del 1929 e 1930 nonostante indicassero (a) in modo preciso i soggetti ai quali si riferivano (fedeli greco-cattolici provenienti dalla Galizia e Transcarpazia) e (b) il luogo della loro nuova residenza (Stati Uniti, Canada e Australia), col tempo vennero applicate anche negli altri territori che non appartenevano alla categoria dei territori di tradizione orientale.

Cosa si deve intendere con il concetto di regioni orientali? La risposta può essere trovata nell'Annuario Pontificio ${ }^{8}$, dove si presenta una breve storia della Congregazione per le Chiese Orientali, o nella pagine ufficiali del sito internet del Vaticano. Questi due fonti indicano che alle regioni orientali appartengono i seguenti Stati: Egitto,

\footnotetext{
${ }^{8}$ Cfr. Annuario Pontificio 2014, Città del Vaticano 2014, 1819.
} 
Eritrea e Nord di Etiopia, Bulgaria, Cipro, Grecia, Iran, Iraq, Libano, Palestina, Siria, Giordania e Turchia. Invece Dimitrio Salachas ${ }^{9}$ a questo elenco aggiunge anche penisola del Sinai, Albania Meridionale e Afghanistan. La estensione a questi Stati del concetto di regioni orientali trova la propria spiegazione nel fatto che secondo l'antica tradizione in questi territori si osservavano i riti orientali ${ }^{10}$.

Occorre anche dire che lo studio del significato del concetto dei territori geografici suscita anche alcune domande. Per esempio, perché la Bulgaria dove il cristianesimo fu diffuso nel IX sec., appartiene alle così dette regioni orientali, invece l'Ucraina, che diventò cristiana un secolo dopo, non si può considerare come una regioni orientali? Lappartenenza a questa categoria dipende dalla divisione politica di quel territorio fatta in passato? Queste domande rimangono aperte e possono diventare oggetto di studio per ricerche successive.

L'analisi scientifica mostra che oltre alle regioni orientali nella prassi ecclesiastica cattolica si applica anche un altro concetto, cioè territori di rito orientale ${ }^{11}$. Secondo la legislazione odierna orientale si tratta innanzitutto di territori geografici che non sono stati elencati tra le regioni orientali e dove il numero dei cristiani orientali prevale

${ }^{9}$ Cfr. D. Salachas, Congregazione per le Chiese Orientali, in: Commento alla Pastor Bonus e alle norme sussidiarie della Curia Romana, a cura di P. V. Pinto. Città del Vaticano 2003, 81-82.

${ }^{10}$ Cfr. M. Malvestiti, La Costituzione Apostolica "Pastor Bonus" e la Congregazione per le Chiese Orientali, Iura Orientalia 10(2014), 88.

${ }^{11}$ Cfr. D. Salachas, Congregazione per le Chiese Orientali..., 82. Seguendo il pensiero dell'Autore i territori di rito orientale sono quelli che non fanno parte del concetto di regioni orientali e sui quali sono stati eretti le circoscrizioni ecclesiastiche proprie delle Chiese Orientali Cattoliche. Nello stesso tempo l'Autore si riferisce al can. $146 \$ 1$ del Codice orientale che dimostra un'altra dimensione dei territori di rito orientale, cioè i territori dove il patriarca o arcivescovo maggiore gode il diritto di erigere le circoscrizioni ecclesiastiche della propria Chiesa sui iuris. Quindi un termine che include due concetti? Secondo il nostro parere è difficile chiamare territori di rito orientale Paesi come Ungheria, Slovacchia dove la maggioranza dei cristiani appartengono alla Chiesa latina, ed invece i fedeli orientali cattolici sono in minoranza numerica. Perciò meglio chiamare territori di rito orientale quelli che vengono interpretati secondo il can. $146 \$ 1$ CCEO. 
sulla presenza dei fedeli appartenenti alle altre Chiese sui iuris (per es. Ucraina). Inoltre questo concetto, cioè territori di rito orientale, è legato alla potestà di giurisdizione del Patriarca o Arcivescovo Maggiore dove il Gerarca ha diritto di erigere circoscrizioni ecclesiastiche come le Metropolie, Eparchie, Esarcati (cfr. can. $146 \$ 1$ CCEO).

Quindi alla categoria di territori di rito orientale, secondo i principi appena elencati, non saranno inclusi le circoscrizioni ecclesiastiche, che nonostante la loro antica origine, per questioni geopolitiche, si trovano nei territori che attualmente fanno parte di un nuovo Stato nel quale prevale la tradizione latina (per es. Chiesa Greco-Cattolica Ucraina in Polonia) e dove non si estende la giurisdizione diretta dell'Arcivescovo Maggiore della Chiesa Ucraina.

L'attività e la vita dei cristiani orientali cattolici e dei fedeli latini situati in un territorio appartenente alle regioni orientali, così come i fedeli orientali cattolici che invece non fanno parte di territori di rito orientale, viene svolta in stretta collaborazione e sostenuta dalla Congregazione per le Chiese Orientali. Invece le questioni ecclesiastiche dei cristiani orientali cattolici appartenenti alla categoria di territori di rito orientale, nella maggioranza dei casi, vengono affidate alla Sezione per i Rapporti con gli Stati del Segretariato di Stato della Sede Apostolica ${ }^{12}$.

\section{La nozione dell' "Ordinariato per fedeli orientali"}

Nel Documento del 2014 della Congregazione per le Chiese Orientali si parla anche di una struttura ecclesiastica organizzata sotto forma di un Ordinariato per i fedeli orientali che appartengono alle Chiese Orientali Cattoliche. La genesi di questa forma di organizzazione ecclesiastica è legata alla presenza dei fedeli orientali cattolici in un certo territorio geografico (nazionale) senza il proprio Gerarca della stessa Chiesa sui iuris. L'Ordinariato è la circoscrizione ecclesiastica eretta dalla Sede Apostolica in alcuni territori nazionali (Stati) della tradizione latina dove si nota un numero elevato di fedeli

\footnotetext{
${ }^{12}$ Cfr. Secretaria Status, Rescriptum ex audientia, 4.01.2006, AAS 98(2006) 65-66.
} 
orientali cattolici di varie Chiese sui iuris. Queste strutture ecclesiastiche vengono erette solo nei casi in cui vi sono determinati motivi per cui non si può creare una struttura gerarchica propria per questi fedeli. Queste questioni possano essere per es.: (a) il numero scarso dei sacerdoti della stessa Chiesa sui iuris; (b) la diffusa presenza dei fedeli nei vari luoghi dello stesso paese. L'Ordinariato, come struttura gerarchica, in principio non è stato previsto dal diritto comune però le sue basi teologiche e giuridiche possono essere trovate nel Decreto sulla missione pastorale dei Vescovi nella Chiesa Christus Dominus $(\operatorname{art.~23})^{13}$.

Anche seguendo la prassi amministrativa della Sede Apostolica si può constatare che l'Ordinariato diventa una forma primaria che dà inizio alla futura organizzazione gerarchica propria per i fedeli di una Chiesa sui iuris. Come esempio ci si può riferire alla situazione dei fedeli greco-cattolici ucraini ${ }^{14}$, fedeli armeni ${ }^{15}$ e della Chiesa maronita ${ }^{16}$ in Francia. Fino al 1960 questi coetus dei fedeli appartenevano ad unico Ordinariato eretto nel 1954 per tutti fedeli orientali sprovvisti della cura pastorale del proprio Gerarca ${ }^{17}$.

L'Ordinariato viene eretto con il decreto emanato dalla Congregazione per le Chiese Orientali e nel suo governo è assoggettato a questo Dicastero della Curia Romana. La particolarità di circoscrizione ecclesiastica si esprime nel fatto che è presieduta dall'Ordinario latino

${ }^{13}$ Cfr. A. Viana, Ordinariato para fieles de ritos orientales, in: Diccionario General de Derecho Canónico, vol. V, a cura di J. Otaduy, A. Viana, J. Sedano, Navarra 2013, 812-814.

${ }^{14}$ Esarcato Apostolico della Chiesa Greco-Cattolica Ucraina in Francia è stato eretto il 22 luglio 1960, invece il 19 gennaio 2013 è stato elevato a livello di eparchia di Saint-Vladimir-le-Grand de Paris - cfr. Annuario Pontificio 2013, 633.

${ }^{15}$ Anche eretto il 22 luglio 1960, invece il 30 giugno 1986 r. modificato come Eparchia Sainte-Croix-de-Paris.

${ }^{16}$ L'Eparchia Notre-Dame du Liban de Paris è stata eretta il 21 luglio 2012.

${ }^{17}$ Sacra Congregatio Pro Ecclesia Orientali, Decretum Ordinariatus pro omnibus Christifidelibus Ritus Orientalis in Gallia degentibus instituitur, 27.07.1954, AAS 47(1955) 612-613. Soвór WATYкAŃski II, Dekret o pasterskich zadaniach biskupów w Kościele Christus Dominus, art. 23 (28.10.1965), in: Ustrój hierarchiczny Kościoła, a cura di W. Kacprzyk, M. Sitarz, Lublin 2006, 95-96. 
che nella maggioranza dei casi è anche il Vescovo diocesano di una diocesi o arcidiocesi latina costituita nel capitale statale ${ }^{18}$. L'Ordinario, oltre alla potestà di governo verso i propri fedeli latini, gode anche della giurisdizione propria e ordinaria verso tutti orientali cattolici che sono stati a lui affidati mediante decreto della propria nomina per questo ufficio ecclesiastico. È utile sottolineare che la giurisdizione che l'Ordinario svolge verso dei fedeli orientali spesso è cumulativa con la potestà dell'Ordinario del luogo dove si trovano fedeli orientali. In più, il 30 aprile 1986, la Congregazione per le Chiese Orientali ha emanato un documento con il quale conferma che qualsiasi atto giuridico verso i fedeli orientali appartenenti ad un Ordinariato, per poter essere considerato valido, deve essere concordato con i relativi Ordinari del luogo ${ }^{19}$.

Tornando al Documento della Congregazione del 2014 nella parte in cui si parla degli obblighi degli Ordinari costituiti per i fedeli orientali cattolici si può affermare che il requisito prescritto, cioè di avvisare la relativa Conferenza Episcopale e la Congregazione per le Chiese Orientali circa l'intenzione di ordinare un candidato sposato diacono o presbitero, è per la liceità e non per la validità di tale atto giuridico.

\section{La situazione attuale}

Nell'ultimo decennio si è attivato molto lo studio circa il ministero pastorale dei ministri orientali cattolici uxorati sia nelle circoscrizioni ecclesiastiche orientali costituite fuori dai propri territori tradizionali che nelle diocesi latine. Il fattore che ha spinto a intraprendere questa ricerca è l'immigrazione di un numero notevole di fedeli orientali cattolici dai propri territori ecclesiastici a nuovi paesi dove la tradizione ecclesiastica è quella latina. Così anche la Sede Apostolica con il Documento del 20 febbraio 2008 nonostante abbia confermato

\footnotetext{
${ }^{18}$ Per esempio in Argentina (Buenos Airos), in Francia (Parigi), in Polonia (Varsavia). Invece è diversa la situazione in Brasile (Rio de Janeiro), Armenia (Gyumri).

${ }^{19}$ Congregazione per le Chiese orientali, "Declaratio" interpretativa del decreto del 27 luglio 1954, 30 aprile 1986, AAS 78(1986) 784-786.
} 
l'obbligatorietà dei propri documenti emanati in passato circa il servizio pastorale dei ministri sacri orientali cattolici in diaspora, ha previsto in certi casi e in situazioni eccezionali una certa deviazione da quelle norme.

Invece il Documento del 14 giugno 2014 emanato dalla Congregazione per le Chiese Orientali cambia notevolmente le disposizioni precedenti espresse nei Decreti del 1929 e 1930. Esse infatti, così come è già stato detto, proibivano ai sacerdoti uxorati di svolgere la pastorale tra i propri fedeli orientali cattolici che si trovavano in diaspora, cioè fuori dei propri territori tradizionali.

Secondo il nuovo ordinamento vi sono le seguenti disposizioni:

1. La concessione del diritto alle strutture gerarchiche delle Chiese Orientali Cattoliche (Metropolie, Eparchie, Esarcati) costituite fuori del territorio della propria Chiesa sui iuris (per esempio della Chiesa Ucraina in USA, Canada, Australia, Germania, Francia, Inghilterra, Polonia) o delle singole circoscrizioni ecclesiastiche non appartenenti a nessuna Chiesa patriarcale o arcivescovile maggiore, o della Chiesa Metropolitana sui iuris come per esempio Chiesa Bulgara, Chiesa Macedone, di ordinare i candidati sposati che sono fedeli della stessa Chiesa sui iuris. Inoltre secondo delle nuove norme il Gerarca orientale prima d'amministrate il sacramento dell'Ordine di diaconato o presbiterato ad un tale candidato deve chiedere per iscritto all'Ordinario latino del luogo della residenza del candidato il suo parere (e non il permesso) e le informazione circa il candidato. Parlando quindi dell'ordinazione dei candidati uxorati in territori fuori della propria tradizione rituale si presume che da questo momento la cura pastorale dei propri fedeli può essere affidata anche ai ministri sacri sposati.

2. La concessione della facoltà di ordinare i candidati uxorati a coloro che presiedono un Ordinariato eretto per i fedeli orientali cattolici sprovvisti del Gerarca proprio. I requisiti che devono essere osservati da parte degli Ordinari prima di compiere tale atto giuridico sono la richiesta di avvisare la relativa Conferenza Episcopale e la Congregazione per le Chiese Orientali. 
3. L'Ordinario del luogo della Chiesa latina nel caso in cui svolge la giurisdizione verso i fedeli orientali cattolici presenti nella propria circoscrizione ecclesiastica, ma sprovvista del proprio Gerarca (per es. la situazione canonica dei fedeli greco-cattolici ucraini in Italia, Spagna, Portogallo) ha pieno diritto di conferire l'ordinazione del diaconato e presbiterato ad un candidato uxorato appartenente alla relativa Chiesa sui iuris. Per effettuare questo diritto il vescovo latino necessita di ottenere il permesso dalla Congregazione per le Chiese Orientali e deve sentire il parere della propria Conferenza Episcopale.

Quindi nei primi due casi il Vescovo orientale e latino per la liceità del proprio atto giuridico (amministrare l'ordinazione del diaconato e presbiterato ad un candidato uxorato) devono informare i relativi uffici ecclesiastici, invece, nel terzo caso, devono ottenere anche il permesso dalla Congregazione per le Chiese Orientali. Poi, nel caso in cui il candidato che appartiene ad una Chiesa orientale cattolica è suddito del ministro della sacra ordinazione della Chiesa latina, il Vescovo latino necessita anche avere un altro permesso speciale da parte della Congregazione per le Chiese orientali (can. $748 \$ 2$ CCEO; can. $1015 \$ 2$ CIC). Invece per evitare queste pratiche burocratiche può tramite le lettere dimissorie chiedere il Vescovo della stessa Chiesa sui iuris alla quale appartiene il candidato di conferire l'ordinazione sacra (can. 747; can. 748 CCEO).

\section{Le questioni da risolvere}

Continuando lo studio del Documento vaticano del 14 giugno 2014 occorre sottolineare il fatto che oltre alla introduzione dei cambiamenti in favore delle Chiese Orientali Cattoliche è necessario segnalare anche altri elementi che richiedono una spiegazione più approfondita.

\section{La classificazione giuridica del Documento}

Ci si chiede qual è il significato giuridico del Documento stesso, cioè a quale categoria di atti amministrativi dell'ambito canonico esso appartiene. 
Per poter dare una risposta scientificamente argomentata occorre fare riferimento ad alcuni documenti che sono in vigore anche oggi e che trattano dell'attività della Curia Romana. Essi sono: la Costituzione Apostolica Pastor Bonus ${ }^{20}$ e il Regolamento Generale della Curia Romana ${ }^{21}$. Secondo il primo documento i Dicasteri vaticani godono esclusivamente della potestà esecutiva ordinaria vicaria e della potestà giudiziaria ${ }^{22}$. Il secondo afferma che questa giurisdizione si realizza tramite l'emanazione di decreti esecutivi generali e di istru$z_{i o n i}{ }^{23}$, che prima della pubblicazione devono ottenere il nulla osta del Sommo Pontefice ${ }^{24}$.

Perciò, nonostante il Documento della Congregazione per le Chiese Orientali del 2014 sia stato pubblicato negli Acta Apostolicae Sedis, viene considerato come atto informativo ${ }^{25}$ che introduce alcuni cambiamenti nelle norme precedenti che regolavano la questione dei sacerdoti orientali uxorati; sulla base di ciò che è stato detto prima, si può affermare quindi che questo Documento può essere considerato come un decreto esecutivo generale per cui non viene studiato nel $\mathrm{CCEO}^{26}$.

\footnotetext{
${ }^{20}$ Cfr. IoAnnis Pauli II, Const. Ap. Pastor Bonus, 28 giungo 1988, AAS 80(1988) 841-930.

${ }^{21}$ Cfr. Giovanni Paolo II, Regolamento Generale della Curia Romana, in: Commento alla Pastor Bonus e alle Norme sussidiarie della Curia Romana, a cura di P. Pinto, Città del Vaticano 2003, 371-471.

${ }^{22}$ Cfr. Pastor Bonus, art. 18, 34-35.

${ }^{23}$ Cfr. Regolamento Generale della Curia Romana, art $125 \$ 2 \ldots, 446$.

${ }^{24}$ Cfr. J. García Martin, Atti amministrativi generali, Roma 2004, 122-126.

${ }^{25}$ Invece i documenti legislativi emanate dal Sede Apostolica per poter essere considerati come le leggi deve non essere pubblicati negli Acta Apostolicae Sedis (can. $1489 \$ 1$ CCEO )

${ }^{26}$ Cfr. A. TANASIYChuK, L'esercitazione della potestà esecutiva nella Chiesa Patriarcale. Caso concreto: can. $82 \$ 1,1$ CCEO, in: La funzione amministrativa nell'ordinamento canonico. Atti del XIV Congresso Internazionale di Diritto Canonico, che è stato celebrato dal (Varsavia, 14-18 settembre 2011), a cura di Józef Wroceński, Marek Stokłosa, Varsavia 2012, 123-130.
} 


\section{La clausola della prima disposizione - informare per iscritto}

Un argomento presente nel Documento che merita di essere studiato più dettagliatamente si trova nella prima disposizione. Riguarda il Gerarca orientale che governa una eparchia o esarcato fuori dal territorio della Chiesa patriarcale o nel territorio dove la tradizione propria è quella latina: prima di poter conferire l'ordinazione del diaconato o del presbiterato al proprio candidato deve per iscritto informare il Vescovo latino del luogo della residenza del candidato.

\section{Prima difficoltà}

Tra le norme del Codice orientale c'è un canone che in modo esplicito dimostra che il fedele per mezzo del domicilio o del quasi-domicilio in un territorio di una parrocchia o di una eparchia/ esarcato ottiene il nuovo Gerarca del luogo e il parroco che sono della stessa Chiesa sui iuris (can. $916 \$ 1$ CCEO). Nel caso in cui il fedele appartiene alle circoscrizioni ecclesiastiche della propria Chiesa sui iuris erette in diaspora, diventa anche soggetto alla giurisdizione del nuovo Gerarca del luogo.

La sua potestà di governo nella maggior parte dei casi è personale, cioè definita non secondo il principio del territorio di una città o di un regione, ma spesso del territorio di un Paese (per esempio Chiesa Greco-Cattolica Ucraina in Germania, Inghilterra o Francia). Tale fedele essendo candidato all'ordinazione sacra nei casi ordinari appartiene ad una certa parrocchia della propria Chiesa sui iuris. In essa ha ottenuto l'educazione cattolica secondo la tradizione della propria Chiesa e nella stesso tempo, per esempio, potrebbe non avere avuto alcun contatto con le realtà parrocchiali che fanno parte di una altra Chiesa sui iuris (Chiesa Ucraina in Polonia, in USA, in Canada, in Australia). In questo caso sorge la domanda: per quali motivi il Vescovo orientale deve per iscritto avvisare l'Ordinario latino?

Le risposte alla domanda possono essere varie, ma si propone di trovare tale risposta tra le norme del Codice orientale.

Una risposta potrebbe essere legata alla prescrizione disciplinare che sottolinea il diritto dato ai responsabili che concedono il permesso al candidato del diaconato o presbiterato, nel caso lo ritengano 
opportuno, di chiedere ad altri Vescovi eparchiali o diocesani il loro parere circa il candidato stesso in quanto in passato risiedeva nel loro territorio (can. $769 \$ 1,6$ CCEO; can. 1051, 2 CIC). È ovvio che nella espressione «territorio» può essere compresa la residenza dentro i confini di un Stato, ma anche fuori del Paese.

Invece una seconda risposta potrebbe essere in relazione alla situazione legata al passaggio ecclesiastico del fedele da una Chiesa sui iuris ad una altra Chiesa: per esempio, un fedele che prima era soggetto alla legislazione latina, dopo aver ricevuto la licenza da parte della Congregazione per le Chiese Orientali, passa ad una Chiesa Orientale Cattolica (can. 32 CCEO; can. $112 \$ 1,1-2$ CIC, Rescritto della Segretaria di Stato dal 26 novembre $\left.1992^{27}\right)^{28}$. Poi, con il tempo, diventa un candidato uxorato per l'ordinazione sacra. La domanda indirizzata al suo Ordinario precedente (Vescovo latino) sarebbe un atto di prudenza e di utilità per i nuovi superiori ecclesiastici, perché il Vescovo del passato potrebbe essere in possesso di alcune informazioni che impediscono l'amministrazione di questo sacramento. Inoltre, per esempio, nel documento di licenza richiesto alla Congregazione per le Chiese Orientali, potrebbe esserci la clausola che proibisca di conferire il sacramento dell'ordine sacro a questa persona.

I due esempi riportati sottolineano il fatto che il Vescovo orientale gode del diritto di cercare le necessarie informazioni circa il candidato, ma non l'obbligo di fare questo. Inoltre viene detto che questa iniziativa deve provenire dal ministro della ordinazione sottolineando nello stesso tempo che questo è un processo informativo che deve avvenire prima dell'ordinazione sacra.

Invece la prescrizione di informare per iscritto, presentata nel Documento della Congregazione, forse occorre considerarla come un obbligo e non come una semplice raccomandazione per il ministro

\footnotetext{
${ }^{27}$ Cfr. Segretaria di Stato, Rescritto "ex audientia SS.mi”, 26.11.1992, AAS 85(1993) 81.

${ }^{28}$ Cfr. A. Tanasiychuk, Il fedele cristiano e il suo stato canonico nella propria Chiesa sui iuris. Questione dell'ascrizione e del passaggio ecclesiastico, Ephemerides Iuris Canonici 52(2011), 303-344.
} 
dell'ordinazione sacra. Perciò sorge la domanda se questa prescrizione non crea un certo conflitto tra stesse norme canoniche perché il Documento non è una legge promulgata dal Supremo Legislatore.

Per poter dare una interpretazione corretta circa questo obbligo prescritto nel Documento si propone di preparare una petizione da parte del Sinodo dei Vescovi della Chiesa patriarcale o arcivescovile maggiore, del Consiglio dei Gerarchi della Chiesa Metropolitana sui iuris o del singolo Vescovo eparchiale al Pontificio Consiglio per Testi Legislativi con la richiesta di interpretazione autentica riguardo a tale prescrizione giuridica.

Seconda difficoltà

Oltre alle questioni puramente giuridiche trattate nel Documento possono essere evidenziate anche altri argomenti che provocano qualche domanda anche di carattere morale da ricondurre tra le questioni che si riferiscono alla protezione del diritto e dignità della persona nei confronti della scelta del proprio stato di vita, cioè sposarsi o rimanere celibe. Nel Documento viene citato il motu proprio di Benedetto XVI Ecclesia in Medio Oriente, che sottolinea il valore notevole del sacerdozio uxorato. Questo stato canonico dei ministri sacri è un elemento antico e proprio delle tradizioni orientali perciò deve essere sostenuto da tutti perché il sacerdote insieme con la propria famiglia è chiamato alla santità tramite un fedele esercizio del servizio sacerdotale che spesso viene fatto in circostanze difficili della vita ${ }^{29}$ (art. 48). Da parte di alcuni candidati all'ordinazione sacra possono sorge la domanda: perché quando si tratta di un candidato uxorato il Vescovo orientale è obbligato ad avvisare in forma scritta l'Ordinario latino e anche di chiedergli le informazione circa il candidato, quando invece nella situazione di candidati celibi è dispensato da tale processo informativo?

\footnotetext{
${ }^{29}$ Cfr. Benedicto XVI, Exhortación Apostólica Postsinodal Ecclesia in Medio Oriente, 14 settembre 2012, art. 48, Città del Vatinano 2012, 46.
} 


\section{Fedeli greco-cattolici ucraini e Ordinari latini}

Un'altra questione che rimane aperta è la pastorale dei fedeli orientali cattolici nei territori dove non sono costituiti le circoscrizioni ecclesiastiche della propria Chiesa sui iuris. Qui si tratta delle situazioni in cui la cura pastorale verso questi fedeli viene svolta dalla gerarchia latina. Come esempi concreti si possono riportare la situazione dei fedeli greco-cattolici ucraini in Italia, Spagna, Portogallo e non solo (anche dei fedeli greco-cattolici rumeni) che sono soggetti alla giurisdizione dei Gerarchi locali latini. Questa situazione rispecchia il can. $916 \$ 5$ CCEO secondo il quale i fedeli cattolici di una Chiesa sui iuris (compresi anche i latini) nel caso in cui sono sprovvisti di propria gerarchia vengono assoggettati al Gerarca del luogo di un altra Chiesa sui iuris.

Tale forma di giurisdizione spesso crea le difficoltà nella organizzazione di una cura pastorale adeguata nei confronti dei fedeli orientali cattolici, come anche può far sorgere nuovi «conflitti giuridici» circa l'applicazione canonica dei due Codici della Chiesa cattolica (CCEO e CIC). Così per esempio spesso accade che le curie diocesane latine nei confronti dei fedeli orientali cattolici applicano la legislazione canonica propria, ossia CIC. Essa sostanzialmente si distingue dalle norme canoniche orientali, poi, è in contrasto nella terminologia giuridica, con lo stato canonico dei ministri sacri e fedeli orientali presenti in questi territori.

Per esempio lo stato canonico delle comunità orientali erette per i fedeli di una Chiesa orientale cattolica in una diocesi latina spesso vengono equiparate alle cappellanie o missioni. La cura pastorale di queste comunità vengono affidate ai sacerdoti orientali che prima hanno ottenuto dal Vescovo diocesano il decreto di nomina per poter svolgere il ministero sacerdotale. Gli atti amministrativi di queste nomine mostrano la varietà formale e terminologica circa le funzioni affidate ai sacerdoti: vicario parrocchiale, cappellano, coadiutore del cappellano, assistente spirituale. Spesso nel decreto di nomina fatta dalla gerarchia ecclesiastica latina vengono citate, al posto delle norme della legislazione orientale alla quale sono assoggettati tutti fedeli 
orientali cattolici, le norme del codice latino (can. 271; can. 564; can. 565; can. 568) $)^{30}$.

Oltre a questo esempio ci possono essere altri esempi in riferimento ad altre norme come quelle che si riferiscono al sacramento del matrimonio, in particolare le prescrizioni circa gli impedimenti che dirimono il matrimonio: l'impedimento di rapimento e trattenimento di una persona con la quale si vuole celebrare il matrimonio (can. 806 CCEO), l'impedimento dell'affinità di secondo grado della linea collaterale (can. $809 \$ 1 \mathrm{CCEO}$ ), l'impedimento della parentela spirituale (can. 811 CCEO). Inoltre, qui occorre ricordare anche le prescrizioni canoniche che si riferiscono alla forma canonica del matrimonio secondo le quali nella celebrazione di questo sacramento devono partecipare esclusivamente il sacerdote o Vescovo (diacono escluso) che amministra questo sacramento al fedele o fedeli appartenenti alla Chiesa orientale cattolica tramite il rito sacro (can. 828 CCEO).

Applicazione del Codice latino nei confronti dei fedeli orientali cattolici non solo è contraria alla stessa legge (can. 1 CIC), ma anche contraddice l'insegnamento del Vaticano II che sottolinea il diritto delle Chiese orientali cattoliche di reggersi secondo la legislazione propria.

\section{Conclusione}

Il Documento della Congregazione per le Chiese Orientali del 14 giungo 2014 non solo fa riferimento ai candidati uxorati per il diaconato e presbiterato presenti nei territori propri della tradizione latina, ma si ritiene che apra anche la possibilità ai sacerdoti sposati orientali cattolici di prestare il loro ministero sacerdotale in questi territori. Questa seconda particolarità è importante perché spesso accadeva che questa categoria dei ministri sacri tramite le prescrizioni particolari di qualche Conferenza Episcopale venivano ostacolati nell'organizzare una adeguata cura pastorale per i propri fedeli risiedenti in diaspora.

\footnotetext{
${ }^{30}$ Cfr. A. Tanasiychuk, Organizzazione della pastorale Greco-Cattolica Ucraina in Italia, in: Cristiani orientali e pastori latini, a cura di P. Gefaell, Giufferé Editore, Milano 2012, 465-477.
} 
Il Documento, che ha introdotto modifiche nella disciplina ecclesiastica, può essere anche considerato come un ulteriore passo nella realizzazione dell'insegnamento del Concilio Vaticano II che nel Decreto per le Chiese Orientali Cattoliche invita al rinnovamento e fedele custodia delle tradizioni proprie di queste Chiese sui iuris ${ }^{31}$.

Inoltre, la pubblicazione di questo Documento continua a dimostrare i cambiamenti che sono avvenuti nelle ecclesiologia cattolica, dopo il Vaticano II, nei confronti dei cristiani orientali che sono in piena comunione con il Romano Pontefice. Non vengono chiamati più in riferimento al rito, ma Chiesa sui iuris (can. 27 CCEO) che secondo il diritto canonico gode di personalità giuridica nella Chiesa universale (can. $921 \$ 2 \mathrm{CCEO}$ ), invece il rito è la sua identità esteriore che si esprime nella liturgia, spiritualità, teologia e disciplina come proprio patrimonio (can. $28 \$ 1 \mathrm{CCEO}$ ).

Questo Documento della Sede Apostolica sottolinea il principio conciliare - ecumenico. Infatti, conservando la tradizione propria delle Chiese Orientali Cattoliche, di ordinare i candidati uxorati fuori del proprio territorio tradizionale e canonico, si dimostra, da parte della Sede Apostolica, non solo il rispetto verso i cristiani appartenenti alle tradizioni orientali che sono in comunione ecclesiastica con essa, ma anche conferma a tutti gli altri cristiani acattolici che è possibile avere «unità nella diversità» (Giovanni Paolo II, Ut unum sint, 1995) ${ }^{32}$.

\footnotetext{
${ }^{31}$ Cfr. Concilium Oecumenicum Vaticanum II, Decretum de Ecclesiis Orientalibus Catholicis Orientalium Ecclesiarum, 21.11.1964, AAS 57(1965) 76-89; tekst polski: Sobór WATYKański II, Dekret o katolickich Kościołach Wschodnich Orientalium Ecclesiarum, in: Sobór Watykański II: Konstytucje, Dekrety, Deklaracje, Pallotinum - Poznań 2002, 169-186, n. 6.

${ }^{32}$ Cfr. Ioannes Paulus II, Litterae encyclicae Ut unum sint: de Oecumenico Officio, AAS 87(1995) 921-982; tekst polski in: JAN PAWEŁ II, Dzieła zebrane, vol. 1, Encykliki, Kraków 2007, 616-666.
} 


\section{Pontificia Praecepta de Clero Uxorato Orientali and the optionality of celibacy in the Eastern Catholic Churches}

The article concerns the document of the Congregation for the Eastern Churches Pontificia Praecepta de Clero Uxorato Orientali published on 14 June 2014, under which outside the traditional Eastern territories within the meaning of the law of the Eastern Catholic Churches, and where the Eastern Catholics reside, the married men can be ordained. The author analyzes the content of the document, explains the terminological problems and its place in the system of canon law, taking up the problematic issues.

Parole Chiave: celibato, diaspora, territori orientali, ordinazione sacerdotale

KEYWORDS: celibacy, diaspora, Eastern territory, sacred ordination

BREVE NOTA SULL'AUTORE:

ANDRIY TANASIYChUK - prezbiter Eparchii Kołomyja-Czerniowce Ukraińskiego Kościoła Greckokatolickiego, wykładowca na Wydziale Prawa Kanonicznego San Pio X w Wenecji. 